\title{
Review: fair evidence supports several tests in investigating fever of unknown origin
} Mourad O, Palda V,Detsky AS. A comprehensive evidence-based approach to fever of unknown origin. Arch Intern Med
2003;163:545-51.

\section{QUESTION: What are the best approaches for a diagnostic workup of fever of unknown origin (FUO)?}

\section{Data sources}

Studies were identified by searching Medline (19662000) using the terms fever of unknown origin, FUO, PUO, and pyrexia of unknown origin; and by reviewing the references of relevant studies.

\section{Study selection}

Studies were selected if they were published in English; they examined the natural history, prognosis, or spectrum of disease associated with FUO, or evaluated a diagnostic test for FUO; the patients had a temperature $>38.3^{\circ} \mathrm{C}$ on several occasions lasting $>3$ weeks; and the diagnosis remained uncertain after 1 week of investigation in hospital. Exclusion criteria were immunosuppression; age $<18$ years; HIV; cancer; or patient groups outside of North America, western Europe, or Scandinavia.

\section{Data extraction}

Data were extracted on the performance and yield of the diagnostic tests. Study quality was assessed according to research design and internal validity (good, fair, or poor).

\section{Main results}

Diagnostic tests, beyond clinical evaluation and basic investigations, for which supporting evidence existed were abdominal computed tomography (CT), nuclear imaging, the Duke criteria for endocarditis, liver biopsy, temporal artery biopsy, and leg Doppler imaging. Test performance, quality rating of the studies, and recommendations for the tests' use are in the table. Bone marrow culture had a low yield $(0-2 \%)$ in 2 fair quality studies. Uncertainty existed for surgical exploration of the abdomen because of the poor quality of the 8 identified studies. No evidence existed for evaluating echocardiography alone, magnetic resonance imaging, bone scan, or D dimer assay. Recommendations were made after considering disease prevalence, test risks and benefits, and strength of supporting studies.

\section{Conclusion}

Fair evidence supports the use of computed tomography, some nuclear imaging tests, the Duke criteria, liver biopsy, temporal artery biopsy, and leg Doppler imaging in the evaluation of fever of unknown origin.

\section{COMMENTARY}

Fever is a cardinal manifestation of disease. When fever is prolonged and its cause is elusive, it becomes a perplexing and frustrating problem for all involved. FUO represents a classic internal medicine problem, considered by many to be the ultimate diagnostic challenge for an astute clinician. ${ }^{1}$

For evidence-based enthusiasts, the "U" in FUO means that no single gold standard exists against which to judge diagnostic tests. So what can we make of an "evidencebased approach?" Given $>200$ possible causes of FUO, an evidence-based algorithm will probably help only in a general sense. Useful information in approaching FUO answers the following questions: What causes are possible (population specific knowledge of the contemporary diseases causing prolonged fever)? ? $^{2}$ What diseases are easily diagnosed or missed with available diagnostic tests? And where do clinicians typically fall short?

The review by Mourad $e t$ al properly indicates that occult disease in the abdomen is still common enough to justify using abdominal CT early, and their review supports the value of considering objective endocarditis criteria for another occult cause of fever. However, as Vanderschueren $e t a l^{\beta}$ observed, with improvements in imaging, bacteriology, and serology over the past 25 years, the real change in distribution of the so called "big three" (infection, cancer, and rheumatological disease) has been a marked increase in the proportion of noninfectious inflammatory diseases causing FUO. Thus, there has been a change from $15-19 \%$ in earlier series to nearly $40 \%$ of diagnosed cases in a more contemporary series. ${ }^{3}$

The methods most likely to be decisive are history and evolution of the condition and a biopsy. A careful history with attention to the evolution of the illness and a willingness to carefully re-examine diagnostic material are good, common sense strategies, reinforced by the realisation that many patients are labelled as FUO because previous clinicians have over interpreted or misinterpreted non-specific findings. This often leads to a biopsy based on objective evidence or a syndromic diagnosis.

Less well known is the fact that because we are better at diagnosing not only cancer, infections, and now non-infectious inflammatory diseases, the prognosis for persons with undiagnosed FUO is better too: $50-100 \%$ recover spontaneously according to Mourad $e t a l$, and $>90 \%$ were well in the recent series by Vanderschueren $e t a l .{ }^{3}$ In Alt and Barker's 1930 series, ${ }^{4}>30 \%$ of those discharged without a diagnosis died, probably from undiagnosed cancer or infectious causes.

Eric B Larson, MD, MPH Group Health Cooperative Seattle, Washington, USA

1 Keefer CS, Leard SE. Prolonged and perplexing fevers. Boston: Little Brown, 1955.

Durack DT, Street AC. Fever of unknown origin - reexamined and redefined. Curr Clin Top Infect Dis 1991;11:35-51

Vanderschueren S, Knockaert D, Adriaenssens T, $e t$ al. From prolonged febrile illness to fever of unknown origin: the challenge continues. Arch Intern Med. 2003;163:1033-41.

4 Alt HL, Barker MH. Fever of unknown origin. JAMA 1930;94:1457-61.

Tests for investigating fever of unknown origin $(F U O)^{*}$

\begin{tabular}{|c|c|c|c|c|}
\hline Tests & Effectiveness & $\begin{array}{l}\text { Level of } \\
\text { evidence }\end{array}$ & $\begin{array}{l}\text { Number and quality } \\
\text { of studies }\end{array}$ & Recommendation \\
\hline Abdominal computed tomography & Sens $71 \%$; Spec $71 \%$ & Fair & 1 fair & Recommended \\
\hline Nuclear imaging & Sens $40-82 \%$; Spec $69-94 \%$ & Fair & 6 fair; 4 poor & $\begin{array}{l}\text { Technetium and indium 111-labeled white blood cell } \\
\text { scans recommended; indium } 111 \mathrm{lgG} \text { and gallium } 67 \\
\text { scans not recommended }\end{array}$ \\
\hline Duke criteria & Spec $99 \%$ & Fair & 1 fair & Recommended \\
\hline Liver biopsy & Diagnostic yield $14-17 \%$ & Fair & 2 fair & Recommended \\
\hline Leg Doppler imaging & & & & $\begin{array}{l}\text { Recommended for diagnosing deep venous } \\
\text { thrombosis, a cause in } 2-6 \% \text { of patients with FUO }\end{array}$ \\
\hline
\end{tabular}

